\title{
El uso de plataformas virtuales y su impacto en el proceso de aprendizaje en las asignaturas de las carreras de Criminología y Ciencias Policiales, de la Universidad Estatal a Distancia de Costa Rica
}

\author{
The Use and Impact of Virtual Platforms in the Learning Process: Experience with Students \\ of Criminology and Police Science at Universidad Estatal a Distancia Costa Rica
}

\section{O uso de plataformas virtuais e seu impacto sobre o processo de aprendizagem nas matérias das carreiras de Criminologia e Ciências Policiais da Universidade Federal a Distância de Costa Rica}

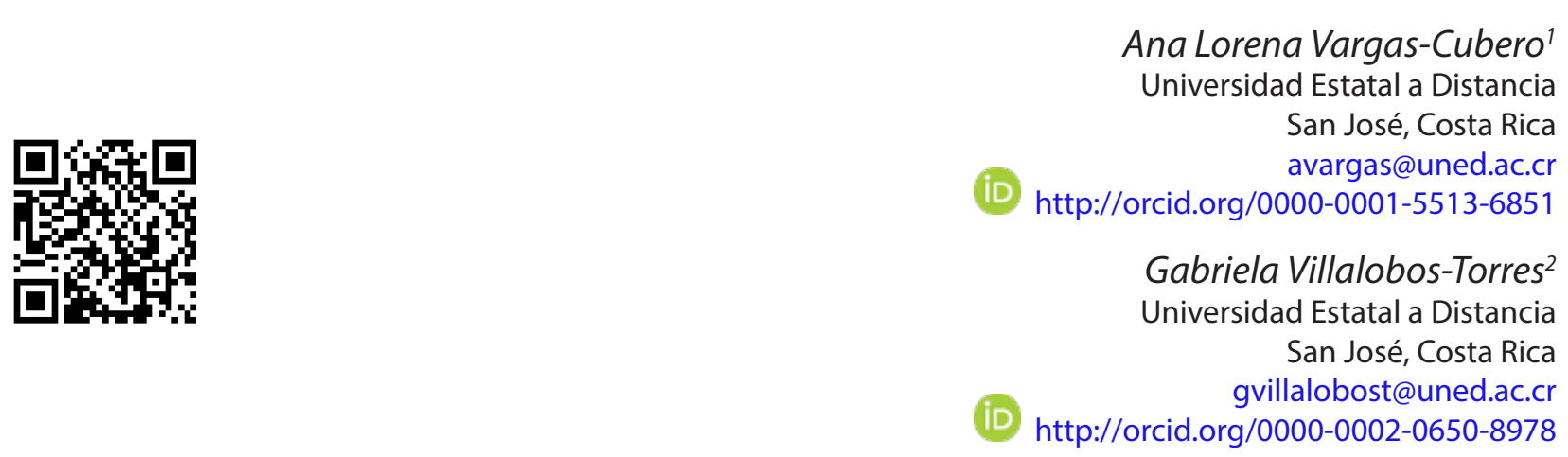

Recibido • Received • Recebido: 06 / 09 / 2016

Corregido • Revised • Revisado: 06 / 07 / 2017

Aceptado • Accepted • Aprovado: 31 / 08 / 2017

\begin{abstract}
${ }^{1}$ Educadora de profesión, especialista en evaluación educativa del Posgrado de la Universidad de Costa Rica. Egresada del Doctorado en Tecnología Instruccional y Educación a Distancia de la NOVA Sowteastern University de la Florida. Ha laborado como educadora en todos los niveles educativos: educación primaria, secundaria y universitaria. Actualmente se desempeña como coordinadora de la Cátedra de Ambiente, Política y Sociedad de la Universidad Estatal a Distancia en la cual labora desde el 2001 año en que se inició como tutora y posteriormente asumió la coordinación de la Cátedra. Ha realizado investigaciones y publicaciones en temáticas propias de las Educación a Distancia y la tecnología como medio de aprendizaje, entre otras temáticas.

${ }^{2}$ Trabajadora Social de profesión, especialista en prevención y atención de la Violencia Intrafamiliar y de Género del Posgrado de la Universidad de Costa Rica y egresada del posgrado en Psicología Laboral de la Universidad Autónoma de Centroamérica. Ha laborado en equipos interdisciplinarios en educación primaria y como profesora en universidades. Actualmente se desempeña como coordinadora de la Cátedra de Trabajo Social de la Universidad Estatal a Distancia, institución a la que ingresó en el año 2009; así mismo es coordinadora del Observatorio Latinoamericano de Trata y Tráfico de Personas Capítulo Costa Rica. Ha realizado investigaciones y publicaciones en temas asociados a la educación a distancia y tecnología para el aprendizaje, violencias, trata de personas y tráfico ilícito de migrantes, entre otros.
\end{abstract}


doi: http://dx.doi.org/10.15359/ree.22-1.2

URL: http://www.una.ac.cr/educare

CORREO: educare@una.cr

Resumen: El objetivo del estudio versa en "determinar de qué forma incide el uso de la plataforma educativa en línea para el aprendizaje en estudiantes de asignaturas de las carreras de Ciencias Criminológicas y Ciencias Policiales", ello por cuanto estos grupos de estudiantes en un alto porcentaje trabajan y estudian, por lo cual un mayor apoyo en cuanto a la mediación, diversificación de recursos y las capacitaciones del aprendizaje en autonomía pueden resultar en mejores condiciones para su aprendizaje. La metodología de investigación tiene un enfoque cuantitativo mediante el modelo de encuesta, pues se aplicó un cuestionario semiestructurado de 19 ítems para explorar aspectos de orden laboral y rendimiento académico, así como los relacionados al desarrollo de la mediación de las asignaturas que cuentan con apoyos virtuales. El instrumento fue aplicado a 50 estudiantes, respondieron 41 de los 5 cursos ofertados por la Cátedra de Ambiente, Política y Sociedad y la Cátedra de Trabajo Social en el I cuatrimestre del 2016 para las carreras antes mencionadas. Posterior a la aplicación del instrumento, la información fue tabulada para realizar el respectivo análisis de datos y establecer las conclusiones pertinentes. Se demostró que el uso de plataformas y de tecnología pueden potenciar y favorecer el aprendizaje, pero por sí misma la tecnología y la plataforma educativa no marcan la diferencia, sino se requieren buena organización de las asignaturas, apoyos y sobre todo mediación adecuada por personal docente capacitado para promover aprendizajes con significatividad.

Palabras claves: Educación a distancia; plataformas educativas; aprendizaje autónomo; aprender a aprender; mediación pedagógica.

\begin{abstract}
The objective of this study is to determine how the use of Moodle online educational platform helps students of Criminology and Police Sciences to learn. A high percentage of the students from these majors work and study facing adverse conditions. Improvements in mediation, variety of resources, and autonomous learning may turn into meaningful learning opportunities for students. The methodology of this study is based on a quantitative approach by means of a sample survey. A nineteen-item semi-structured questionnaire was administered to fifty students to explore aspects related to working order, academic performance, and online course mediation. Forty-one out of fifty participants responded evaluating two courses from the Cátedra de Ambiente, Política y Sociedad [Chair of Environment, Politics, and Society] and three courses from the Cátedra de Trabajo Social [Chair of Social Work] that were offered during the first quarter of 2016. The gathered information was tabulated to facilitate its analysis. The results indicate that the use of online platforms and technology can be used to improve meaningful learning. However, the resources do not have an incidence without well-structured courses, resources, and the appropriate mediation from instructors.
\end{abstract}

Keywords: Distance education; educational platforms; autonomous learning; learn to learn; pedagogical mediation.

Resumo: O objetivo do estudo é "determinar a influencia do uso da plataforma de educação online nos estudantes em seu processo de aprendizagem, das matérias da carreira de Criminologia e Ciências Policiais", isso porque a maioria destes estudantes trabalha e estuda ao mesmo tempo, de modo que, recebendo mais ajuda em termos de mediação, diversificação dos recursos e capacitação para aprender de forma independente, pode resultar em melhores condições para sua própria aprendizagem. A metodologia desta pesquisa tem uma abordagem quantitativa, usando o modelo de entrevista semiestruturada contendo 19 perguntas, aplicado para explorar os 


\begin{abstract}
aspectos relacionados com o trabalho e desempenho académico, bem como outros relacionados com a mediação das matérias que são aplicadas com ajuda virtual. O questionário foi aplicado a 50 estudantes, os quais responderam 41 estudantes, participantes dos 5 cursos oferecidos pelo Departamento de Meio Ambiente, Política e Sociedade e pelo Departamento de Serviço Social, nos primeiros quatro meses de 2016, das carreiras mencionadas. Depois da aplicação do questionário, as informações foram tabuladas para a respectiva análise de dados e estabelecer as conclusões adequadas. Foi demonstrado que o uso de plataformas e tecnologia pode melhorar e promover a aprendizagem, mas por si só a tecnologia e a plataforma educacional não fazem a diferença, porém uma boa organização de cursos, apoios, e sobretudo a mediação apropriada por parte dos professores treinados podem promover aprendizagens significativas.
\end{abstract}

Palavras-chave: Educação a distância; plataformas educacionais; auto-aprendizagem; aprender a aprender; mediação pedagógica

\title{
Introducción
}

La Universidad Estatal a Distancia (UNED) de Costa Rica plantea un modelo educativo a distancia dondeactualmenteofreceasignaturastanto a distanciatradicional comootrasapoyadas en medios tecnológicos y en plataformas virtuales para la educación. Independientemente de los apoyos tecnológicos que ofrezca, la educación a distancia procura el aprendizaje individual o grupal a través de un diálogo didáctico mediado entre docentes y estudiantes desde espacios físicos distintos (García-Aretio, 2014).

Dado que se ofertan diferentes modalidades de asignaturas a distancia, surge como motivo de estudio el cómo influyen esas particularidades educativas en el proceso de aprendizaje del estudiantado. Concretamente de las personas que cursan las carreras de Criminología y Ciencias Policiales, lo anterior debido a que varias de estas personas se encuentran trabajando en áreas relacionadas con esas carreras (UNED, 2016) en las que las demandas de horario y jornada laboral son poco convencionales al trabajar por turnos, en lugares alejados, feriados y fines de semana, operativos nocturnos y en labores peligrosas y estresantes (Smith y Alvarez, 2007).

Por otro lado, quienes no laboran aún también se enfrentan distintas tipologías de cursos en la carrera y se hace necesario dilucidar cuál les favorece mejor para su proceso de aprendizaje, dado que la UNED, por su modelo educativo, en principio, ofrece mayores posibilidades de estudios profesionales y culminación de estos a distintos sectores que por razones de tiempo, laborales, familiares $u$ otros se les dificulte estarse trasladando hasta un centro educativo para formarse académicamente. Por lo que se buscará investigar, mediante encuestas de carácter mixto con estudiantes de esas carreras, cómo incide la modalidad de asignatura a distancia en su proceso de aprendizaje. 
doi: http://dx.doi.org/10.15359/ree.22-1.2

URL: http://www.una.ac.cr/educare

CORREO: educare@una.cr

\section{Referente teórico}

Los referentes teóricos que sustentan esta investigación están relacionados con conceptos concernientes al modelo educativo a distancia, las plataformas educativas en línea y su adecuada mediación pedagógica, las características propias del aprendizaje a distancia como un proceso de autonomía y autorregulación y los factores que puedan promover el aprendizaje desde estas plataformas.

Varias personas autoras han propuesto una definición de educación a distancia, una de las más clásicas es la planteada por García-Aretio (1987), quien la define en los siguientes términos:

Un sistema tecnológico de comunicación [de masas] y bidireccional, que sustituye la interacción ... en el aula de profesor y alumno como medio [preferencial] de enseñanza, por la acción sistemática y conjunta de diversos recursos didácticos y el apoyo de una organización tutorial, que propician el aprendizaje autónomo de los estudiantes. (p. 8)

La educación a distancia ha pasado por varios periodos y ha evolucionado desde aquella sostenida por un sistema de correo convencional a uno apoyado en medios tecnológicos y multimedial, donde la interacción ya no es bidireccional sino que permite la comunicación multidireccional entre docente-estudiante y estudiante- estudiante (Cooperberg, 2002).

En este sentido, en los últimos años, la Universidad en que se realizó el estudio ha venido incursionando en el uso de plataformas educativas en línea, las cuales son, según Coates et al. (2005, citados por Dans, 2009):

Herramientas estandarizadas, o de desarrollo propio, para llevar a cabo la interacción entre los diversos participantes en el proceso (profesorado, alumnos, supervisores, tutores, encargados de administración, etc.); dichas herramientas son conocidas como Learning Management Systems o LMS. Las citadas plataformas o LMS ofrecen a los alumnos un entorno cerrado y controlado en el que las instituciones académicas pueden establecer elementos correspondientes a funciones, tales como foros de participación, herramientas de retroalimentación, módulos educativos de contenidos, mecanismos de comunicación, etc., intentando proporcionar un ambiente lo más uniforme y familiar posible. (p. 2)

Así, el uso de estas plataformas puede potenciar el aprovechamiento de una serie de recursos y herramientas antes no tan fácilmente disponibles para el estudiantado tales como materiales digitales, multimedia, espacios colaborativos y cooperativos de aprendizaje conjunto para estudiantes a distancia y, por otro lado, la posibilidad de interacción entre docentes-estudiantes y estudiantes-estudiantes, con lo que generan espacios y oportunidades de aprendizaje en un modelo educativo a distancia (Sánchez, 2009). 
Al respecto, el aprendizaje que se busca potenciar en un modelo educativo a distancia es aquel que sea autónomo y autorregulado, para favorecer el logro de aprendizajes significativos, la metacognición y el éxito académico entre otros aspectos, lo que puede verse en estudiantes que aprenden de manera autónoma.

Según Manrique (2004), esta debe ser la principal meta de la educación y se expresa en aprender a aprender. Con la autonomía en el aprendizaje se pretende que la persona que aprende sea capaz de gestionar su propio conocimiento de modo que logre no solo aprendizajes significativos, sino también que sea un estudiantado exitoso. Para ello se supone la generación de un esquema que le permita el control mental, emocional, de tiempo, de recursos, de solicitud de ayudas y de uso de estrategias pertinentes con el fin de alcanzar el éxito en el aprendizaje. La interacción con sus pares y docentes es fundamental y con ello el aprender a aprender, lo que conlleva a la toma de decisiones más acertadas para regular el propio aprendizaje (Monereo, 2008; Monereo y Barberá, 2000).

El aprender-aprender es esencial en la educación a distancia y propio de estudiantes que se autorregulan, esto es, una persona que estudia no como un ente pasivo sino por el contrario activo y constructivo, de manera que es capaz de controlar, supervisar y regular determinados aspectos de su conocimiento, motivación y comportamiento y de ciertas características del contexto, ello mediante el uso de estrategias que le permiten de mejor manera el logro de las metas de aprendizaje propuestas (Pintrich, 2000; Piñeiro, Valle, González y Rodríguez, 2002).

Este tipo de aprendizaje no es una habilidad innata, sino que es un proceso en el que están presentes diversas variables como lo metacognitivo, lo motivacional y la percepción de autoeficacia, encaminados al logro de aprendizajes que podrán ser utilizados en otros escenarios (Lamas, 2008). Cuando el estudiantado ha aprendido a aprender puede apoyarse en experiencias relevantes de aprendizajes anteriores, con el fin de tener éxito y lograr los objetivos planteados, así como de utilizar y aplicar los nuevos conocimientos y capacidades en otros contextos (Comisión Europea, 2006).

Por otra parte, el uso de las TIC para el aprendizaje ha tenido distintas concepciones, desde quienes asumen que la tecnología en sí misma es una herramienta para el aprendizaje, hasta quienes como Clark (2001) han planteado que la tecnología es como el camión que lleva los helados, en el sentido de que la tecnología en sí misma no marca una diferencia en la mejora del proceso de aprendizaje, sino que debe ser mediada de tal modo que sea útil como herramienta para ese fin.

No obstante, en este sentido, lo que cobra importancia no es la tecnología en sí, tal y como se desprende de la siguiente cita: 
doi: http://dx.doi.org/10.15359/ree.22-1.2

URL: http://www.una.ac.cr/educare

CORREO: educare@una.cr

Existe unanimidad en aceptar que las grandes aportaciones de las tecnologías (informática, telemática y multimedia) se relacionan sobre todo con el fácil acceso a fuentes de información, con el procesamiento de datos y con la comunicación inmediata, sincrónica y asincrónica con otras personas. De tal manera que, en la actualidad, el acceso a la información ya no es la cuestión que preocupa, sino la gestión de los distintos saberes y cómo fomentar el deseo de saber frente a la sobreinformación circundante en la sociedad y cómo formar los marcos de referencia para procesar la información disponible. (Rodríguez, 2010, p. 34)

Así, para esta investigación se parte de la noción de tecnología como una herramienta que puede contribuir al aprendizaje en tanto esté mediada de manera adecuada, de modo tal que quien estudia encuentre en ella un medio más y no un fin en sí misma.

Para ello, el modelo educativo presenta diversos elementos que intervienen en distintas formas en la mediación pedagógica que se practica:

- Separación docente-estudiante

- Utilización de medios técnicos

- Organización de apoyo-tutoría

- Aprendizaje independiente y flexible

- Comunicación bidireccional

- Enfoque tecnológico

- Comunicación masiva (Pagano, 2007).

De los elementos anteriores se puede inferir que en este modelo hay aspectos que favorecen el aprendizaje autónomo y flexible, la meta de aprender a aprender, y para ello se apoyan de una persona tutora orientadora, de medios idóneos y de medios tecnológicos en los que se busca que el estudiantado use esas herramientas en su proceso de aprendizaje.

La forma como se haga uso de esas herramientas, como se dé la mediación entre el contenido, las metas de aprendizajes y quienes aprenden resultan elementales en el proceso de aprendizaje.

Para esta investigación interesa resaltar el tema de la investigación en los medios virtuales, y es que la incorporación de las TIC a la educación a distancia y de las plataformas educativas en línea han generado otras formas de interactuar entre los sujetos de aprendizaje, donde incluso ha surgido la noción de contexto virtual, que considera la globalidad de las acciones de enseñanza 
y de aprendizaje virtual. Así se deben tener en cuenta aspectos como las distintas relaciones e interacciones generadoras de microcontextos educativos configurados por diferentes actores y su interacción, en la que se puede ver la mediación desde distintas perspectivas como lo son el personal docente y el soporte tecnológico que en ese proceso aportan, para marcar un escenario de enseñanza y de aprendizaje virtual.

\section{Propósito}

Este estudio se plantea con el fin de valorar la incidencia del uso de plataformas educativas en línea y el favorecimiento en el proceso de aprendizaje en estudiantes -la mayoría personas trabajadoras- de las carreras de Ciencias Criminológicas y Ciencias Policiales pertenecientes a la Escuela de Ciencias Sociales y Humanidades de la UNED.

Ello, en tanto que la población de estudiantes trabajadores y trabajadoras de estas carreras se enfrentan a dificultosos horarios y extensas jornadas laborales, además de sus demandas personales y familiares, por lo que es importante conocer, a partir de la flexibilidad horaria y la a sincronía que ofrecen las asignaturas con apoyo en la plataforma educativa, cómo influyen en el proceso de aprendizaje la modalidad de educación distancia de asignaturas híbridas o $100 \%$ en línea frente a aquellas que aún presentan componentes de educación a distancia más tradicional.

Se busca determinar entonces de qué forma inciden el uso de la plataforma educativa en línea para el aprendizaje en estudiantes de asignaturas de las carreras de Ciencias Criminológicas y Ciencias Policiales.

\section{Metodología}

Para el estudio se tomó en cuenta la totalidad de la población (50 estudiantes) que actualmente están cursando asignaturas ofertadas por las Cátedras de Ambiente, Política y Sociedad ( 2 cursos: 20 estudiantes) y Trabajo Social (3 cursos: 30 estudiantes) con algún componente en línea de las Carreras de Bachillerato en Ciencias Criminológicas y Bachillerato en Ciencias Policiales, ya sean totalmente en línea o bajo la modalidad híbrida.

A estos grupos de estudiantes se les solicitó completar el instrumento de recolección de la información, de los cuales 41 completaron lo solicitado: 15 mujeres y 26 varones, número por sexo que concuerda con los porcentajes de matrícula de estudiantes de la UNED, en donde para el año 2015 la matrícula total en estas Carreras fue de 388 estudiantes, el 60\% de la matrícula en ambas carreras corresponde a hombres (UNED, 2016). 
doi: http://dx.doi.org/10.15359/ree.22-1.2

URL: http://www.una.ac.cr/educare

CORREO: educare@una.cr

Tal como se mencionó, el contexto de estas personas es que quienes estudian se enfrentan a jornadas laborales de tiempo completo, en horarios rotativos y trabajando domingos y feriados, lo que lleva a mayores dificultades para cursar estudios en una sistema sincrónico y presencial. También hay personas no trabajadoras que igualmente eligieron el modelo educativo de la UNED, el cual les presenta mayores flexibilidades de estudio y tiempo frente a otros modelos presenciales.

La metodología de investigación seguida tiene un enfoque cuantitativo mediante el modelo de encuesta, pues se aplicó un cuestionario semiestructurado, según lo define Hernández, Fernández y Baptista (2014) como un conjunto de preguntas basadas en variables que se pretenden medir, las cuales están asociadas al favorecimiento del éxito académico de estudiantes, dependiendo de la modalidad de curso a distancia ya sea tradicional, híbrida o totalmente en línea.

Dicho cuestionario semiestructurado se compuso de 19ítems, les fue enviado en línea (para que pudieran responder el mismo cuando tuvieren oportunidad de hacerlo) con la intención de explorar aspectos de orden laboral y de rendimiento académico estudiantil y elementos propios del desarrollo de la mediación de las asignaturas que cuentan con apoyos virtuales. Esto, con el fin de determinar cómo favorece el aprendizaje de estudiantes aquellas materias que cuentan con apoyo en las plataformas virtuales o que se gestionan totalmente en esos entornos, frente a las que no lo tienen y se desarrollan en un sistema de distancia tradicional.

El estudiantado fue contactado mediante la plataforma en línea de la Universidad en el I cuatrimestre del 2016 para que completaran el instrumento. Posteriormente se tabuló la información y se realizó el análisis a la luz de los datos recolectados, los cuales se graficaron y se fueron comparando con teoría relativa al tema, con el fin de visualizar lo planteado por los sujetos participantes del estudio y así formular conclusiones pertinentes a la problemática expuesta.

\section{Resultados: Presentación de datos y análisis}

Tomando como base los resultados del análisis de la información recopilada, se puede citar que, de la totalidad de personas que respondieron el instrumento, 14 solo estudian (8 mujeres y 6 hombres) y 27 estudian y trabajan (7 mujeres y 20 hombres), dividiéndose en 30 en la Carrera de Ciencias Policiales y 11 en Ciencias Criminológicas. Se desempeñan en labores relacionadas con sus estudios en lugares como el Ministerio de Seguridad Pública, Ministerio de Obras Públicas y Transportes, Ministerio de Justicia y Gracia, Poder Judicial, Ministerio de la Presidencia y en Seguridad Privada; ejercen funciones como agentes de policía, policía de tránsito, policía de control fiscal, policía carcelaria o agentes de seguridad privada. Así mismo, trabajan a tiempo completo en jornadas laborales diurnas, nocturnas y mixtas y con horarios

Ana Lorena Vargas-Cubero y Gabriela Villalobos-Torres

Los artículos de la Revista Electrónica Educare del Centro de Investigación y Docencia en Educación de la Universidad Nacional, Costa Rica, se comparten bajo términos de la Licencia Creative Commons: Reconocimiento, No Comercial, Sin Obra Derivada 3.0 Costa Rica. Las autorizaciones adicionales a las aquí delimitadas se pueden obtener en el correo: educare@una.cr 
variables, en ocasiones 7 días trabajados por 7 días libres, dos días trabajados por dos días libres, seis laborados por seis de descanso o por periodos de entre 10 y 12 horas diarias, lo que hace que sus jornadas sean extenuantes y que el tiempo libre sea repartido en múltiples actividades que van desde lo personal, académico, recreativo y familiar.

Por lo anterior, la educación a distancia y el uso de las plataformas educativas en línea se convierten en la alternativa para cursar estudios superiores para personas con características como las señaladas en el acápite anterior. En tanto este modelo educativo facilita el acceso a estudios universitarios, al proponer una comunicación mediada de doble vía entre profesorado formador y estudiantes y, en algunos casos, de estudiantes entre sí a través de diferentes medios. A la vez, quienes estudian cuentan con el soporte de una institución que apoya el proceso mediante la planificación y diseño de las carreras y cursos, la producción de materiales y otros recursos, así como la evaluación y el seguimiento del proceso de aprendizaje del alumnado (García-Aretio, 2001).

Estudios como los de Axelbred y Valle (1981), Cooper, Davidson y Robinson (1982), Kroes (1985) y Reese (1986) mencionan que los niveles de estrés que experimentan las personas que trabajan como policías o en seguridad son mayores a los sufridos por otros profesionales, tesis a la que se suman Brown y Campbell (1994) quienes clasifican las fuentes de riesgo de estrés en cuatro grupos: organizaciones o de gestión, inherentes al desempeño de tareas, relacionados con la comunidad y los relacionados con el sistema judicial, donde es catalogado el trabajo policial como una ocupación inherentemente estresante.

El Instituto Nacional para para la Seguridad y Salud Ocupacional, NIOSH (2015) de los Estados Unidos menciona como causas de estrés, entre otras, los diseños de los trabajos, es decir, jornadas largas, extenuantes y trabajos frenéticos, altos niveles de responsabilidad y demasiadas funciones, pocas horas de descanso, falta de oportunidades para el crecimiento personal, las cuales están presentes en el estudiantado que labora en seguridad y estudia a la vez.

Por otra parte, de la totalidad de estudiantes de la muestra, el 71\% tiene personas que dependen económicamente de ellos y ellas, hijos, hijas o cónyuge principalmente, lo que contribuye a aumentar el nivel de responsabilidades personales que deben asumir.

A nivel de estudiante UNED, en la mayoría de los casos no matriculan los bloques completos de asignaturas, así que el promedio es 3 materias por cuatrimestre generalmente las aprueba. Cuando pierden una asignatura las razones principales son excesiva carga laboral que les impide cumplir con los trabajos asignados y estudiar lo suficiente, inadecuado manejo de los tiempos o situaciones inesperadas a nivel de salud o con el equipo de cómputo o el acceso a internet.

La totalidad de estudiantes han matriculado a lo largo de sus estudios universitarios asignaturas en las tres modalidades que oferta la Universidad: tradicional a distancia, híbrida y $100 \%$ virtual, por lo que tienen experiencia en cada una de ellas. 
doi: http://dx.doi.org/10.15359/ree.22-1.2

URL: http://www.una.ac.cr/educare

CORREO: educare@una.cr

En la Figura 1 se puede observar la preferencia del estudiantado por cursar estas asignaturas:

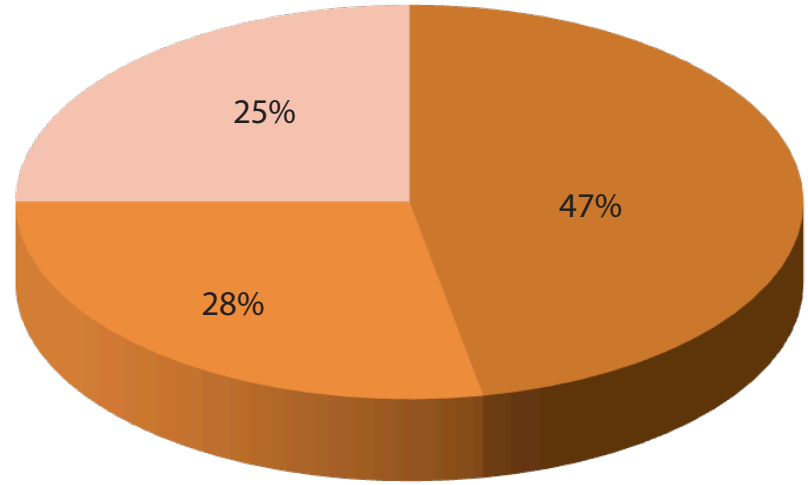

En línea (100\% virtual)

Híbrida

A distancia tradicional

Figura 1: Preferencia del estudiantado por cursar las distintas modalidades de asignaturas en el modelo educativo a distancia.

Nota: Elaboración propia, a partir del cuestionario aplicado al estudiantado que cursa asignaturas en línea.

El estudiantado considera las asignaturas 100\% virtuales más accesibles, con actividades dinámicas adecuadas a sus carreras (54\%), la materia más comprensible lo que les permite un mayor avance en sus estudios con mejores calificaciones (51\%) y un mayor acompañamiento por parte del profesorado de las asignaturas (51\%).

Estos señalamientos son similares con las materias híbridas (28\% las privilegia), al tener la oportunidad de relacionarse con sus compañeros y compañeras a través de la plataforma y a los beneficios que se obtienen mediante la mediación pedagógica que se ofrece con los entornos virtuales (83\%).

El $90 \%$ menciona que en estas dos modalidades las tareas se adecúan más a las carreras que se cursan, mejor acceso a los materiales (88\%), mayor aprendizaje (88\%), mejor mediación a través del seguimiento por parte de docentes $(83 \%$ y y posibilidades de avanzar en sus carreras sin perder asignaturas (85\%).

Lo anterior es concordante con la posibilidad que se plantea en estudiantado a distancia de generar un aprendizaje autónomo y autorregulado apoyándose en experiencias previas para distribuir sus tiempos, así como en la interacción con docentes y pares para alcanzar los objetivos de aprendizaje. , donde el estudiantado se torna en un autogenerador de pensamientos, sentimientos y conductas que se orientan a la consecución de las metas educativas (Zimmerman, 2002). 
En cuanto a las asignaturas que se ofrecen de forma tradicional, el $25 \%$ destacan la posibilidad de organizarse en el cuatrimestre, ya que desde un inicio conocen la totalidad de trabajos que deben presentar y la forma de hacerlos, así como el acceso directo a los materiales al recibirlos en forma física al inicio del periodo cursado (29\%), lo que les facilita el estudiar en cualquier lugar y tener la oportunidad de asistir a tutorías, que se convierten en espacios de análisis de contenidos temáticos, realimentación y orientación para la elaboración de sus proyectos o tareas.

El punto que se rescata, en mayor medida, es que a través de la presencialidad han logrado obtener buenas calificaciones (24\% de estudiantes), lo que con las asignaturas híbridas se les dificulta (solo el $15 \%$ ha alcanzado mejores calificaciones en esta modalidad), ya que distribuyen su tiempo desde el inicio y saben de antemano qué es lo que deben entregar y en cuál fecha, mientras que de forma híbrida deben realizar exámenes presenciales y actividades en la plataforma, lo que consideran como un recargo de trabajo.

Mención aparte es señalar que 37\% de estudiantes prefieren asignaturas en modalidad tradicional y $63 \%$ en modalidad completamente virtual por su situación personal, citan en cada caso que estos se ajustan a sus horarios y jornadas laborales, en especial cuando deben asumir dobles y triples jornadas como madres-padres, estudiantes y personas trabajadoras, ya que en momentos de descanso o por las noches estudian y cumplen con las actividades solicitadas o recargan su tiempo académico en los días de descanso o libres, aunque estos no siempre concuerdan con fechas de entrega de trabajos en la plataforma.

Es importante destacar, en ese punto, lo mencionado por Carrillo y Ríos (2013) en la investigación Trabajo y rendimiento escolar de los estudiantes universitarios. El caso de la Universidad de Guadalajara, México, cuyo análisis versó en el impacto de la jornada laboral sobre el rendimiento escolar del estudiantado de licenciatura de la Universidad de Guadalajara, tomando en consideración que este centro universitario oferta carreras de manera presencial, las cuales se podrían correlacionar con las asignaturas ofertadas por la UNED de forma tradicional.

De las conclusiones de la investigación (Carrillo y Ríos, 2013) se desprenden las siguientes:

1. En estudios realizados en varios países se observa que ciertos tipos de ocupaciones -o de jornadas largas- impactan negativamente el rendimiento escolar....

2. En la percepción de las dos terceras partes de los estudiantes universitarios que trabajan [muestra de su investigación], su ocupación laboral les reduce el tiempo disponible para las actividades escolares y personales y les afecta negativamente en el rendimiento académico. (pp. 31-32) 
doi: http://dx.doi.org/10.15359/ree.22-1.2

URL: http://www.una.ac.cr/educare

CORREO: educare@una.cr

Resultados similares se observan en la investigación de García (2015) Percepción docente del rendimiento académico de los estudiantes universitarios de la USAC Guatemala que trabajan y estudian al mencionar "que para los docentes [de la Universidad de San Carlos] la jornada laboral de los estudiantes universitarios sumada a la jornada de estudio, ... influyen negativamente sobre el rendimiento académico de los estudiantes universitarios (p. 13).

Lo anterior se coteja con la precepción del estudiantado de la UNED anteriormente mencionada, a lo que se debe añadir que el tipo de labores que ejecutan estas personas junto con sus horarios son factores aún más determinantes en la aprobación o no de asignaturas y en el nivel promedio de calificación obtenida al finalizarla.

Tomando en cuenta los aspectos señalados anteriormente, se le consultó al estudiantado si las asignaturas que poseen componentes virtuales le han ayudado a tener mayor éxito académico en comparación con aquellas que no lo tienen, al considerar las particularidades que tienen al desempeñarse laboralmente en ocupaciones con jornadas de trabajo extensivas, en jornadas mixtas o diurnas y con responsabilidades familiares que asumir (ver Figura 2).

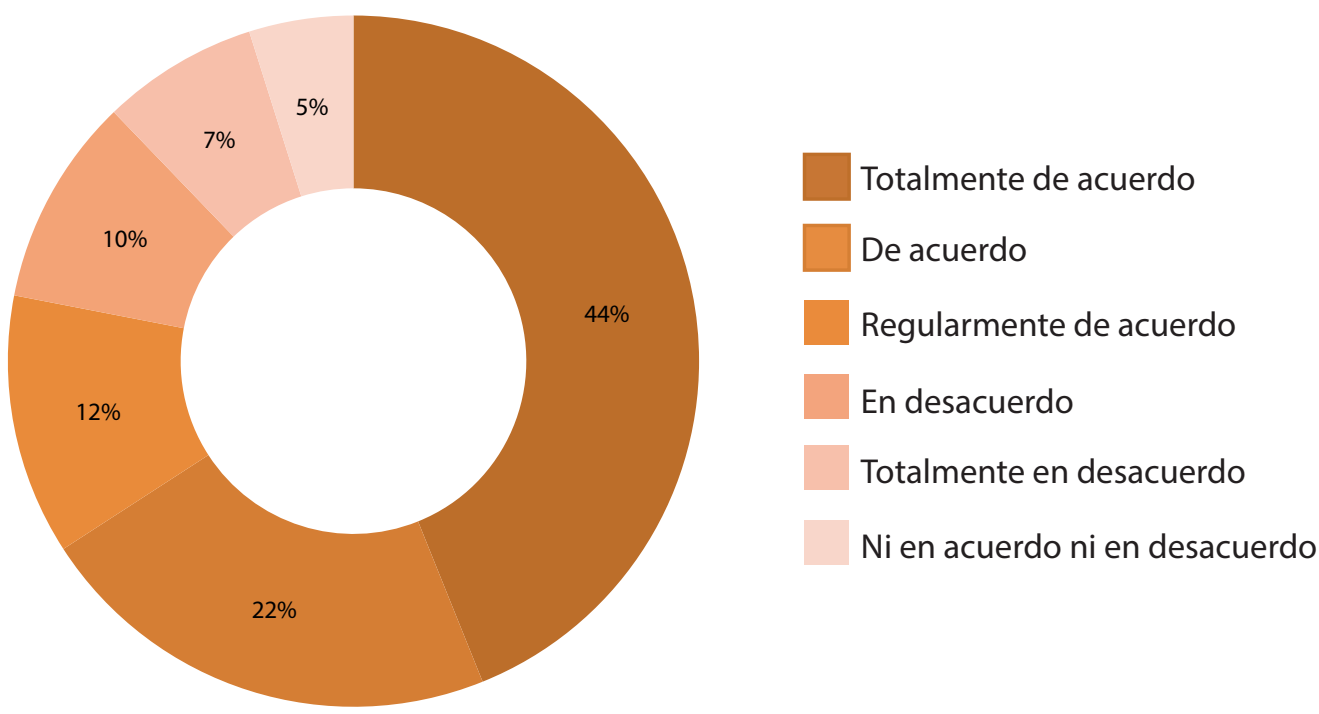

Figura 2: Éxito académico mediante asignaturas que poseen componentes virtuales Universidad Estatal a Distancia I cuatrimestre 2016.

Nota: Elaboración propia, a partir del cuestionario aplicado al estudiantado que cursa asignaturas en línea.

Las asignaturas con componentes virtuales permiten potencializar el uso de las TIC, ya que pueden descargar, desde cualquier lugar con acceso a internet, documentos adicionales, multimedios y otras herramientas que les facilitan el aprender a aprender el cual es, según la Universidad en la que se realizó el estudio, el objetivo más ambicioso e irrenunciable de la educación 
a distancia. Ello corresponde a que quien aprende pueda construir aprendizajes significativos por sí en una amplia gama de situaciones y circunstancias (UNED, Consejo Universitario, 2004) logrando, a su vez, discernir y seleccionar la información correcta para su formación académica y la aprehensión de conocimientos de la sobreinformación existente en la sociedad.

Entre las razones que cita el estudiantado para esa preferencia están las mencionadas en la Figura 3.

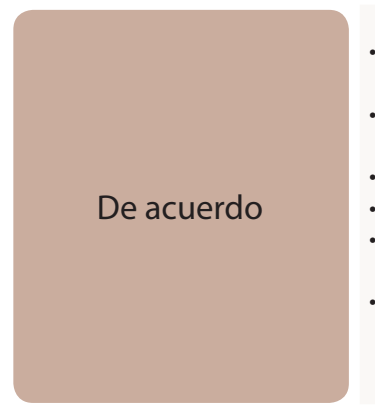

- Mayor contacto entre profesor(a)-estudiante, lo que favorece las prontas respuestas a interrogantes y dudas, así como a promover el compañerismo entre estudiantes.

- Se ingresa a la plataforma a toda hora y desde cualquier lugar por lo que se ajusta a sus horarios laborales. Se descarga la información desde una computadora o desde el celular.

- La materia se puede estar actualizando por lo que se aprende con lo más reciente.

- Las actividades que se proponen pueden ser más creativas y diversas.

- La entrega de tareas se hace en un periodo más extenso de tiempo, no implica desplazamiento para ello, reflejándose en un ahorro en tiempo y dinero.

- La modalidad híbrida permite obtener el beneficio de ambas partes: los exámenes para medir el nivel de comprensión de los temas y la oportunidad de un seguimiento y apoyo mediante la plataforma.

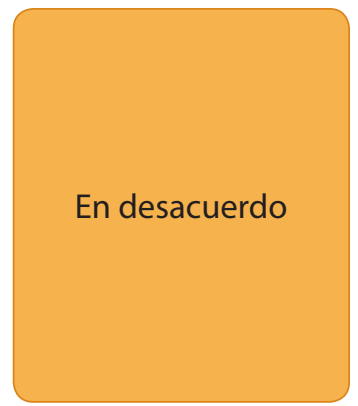

- Las tutorías presenciales permiten un contacto cara a cara entre docente y estudiantes, se satisfacen dudas y se apoya directamente para los trabajos a presentar.

- No siempre se cuenta con acceso a internet o se cuenta con apoyo tecnolólgico apropiado.

- La plataforma no funciona y eso impide descargar materiales o subir trabajos.

- El material impreso favorece el poder estudiar en pequeños momentos y en diferentes lugares, exista o no conexión a internet.

- De forma virtual en ocasiones suben demasiada información, alguna desactualizada o no apropiada al contexto y realiad nacional, lo que entorpece la asimilación de la misma y el aprovechamiento para el conocimiento.

- Las modalidades tradicionales permite tener mayor claridad en la entrega de trabajos y contenidos de examen, la guía es mejor y se conoce desde un inicio. En modalidad virtual la carga es mayor al estar haciendo trabajos semanalmente.

Figura 3: Motivaciones para la preferencia de asignaturas con componentes virtuales en la Universidad Estatal a Distancia I cuatrimestre 2016.

Nota: Elaboración propia, a partir de cuestionario aplicado a estudiantes que cursan asignaturas en línea.

Estas respuestas concuerdan con las opiniones sobre la preferencia de las modalidades de enseñanza-aprendizaje de la UNED, en la que el estudiantado expresa que de la modalidad tradicional les gusta el poder estar sin el estrés de revisar la plataforma y subir trabajos, la practicidad de que en la Orientación de la asignatura se le brinda el detalle de contenidos 
doi: http://dx.doi.org/10.15359/ree.22-1.2

URL: http://www.una.ac.cr/educare

CORREO: educare@una.cr

evaluativos y formativos de esta, se puede estudiar a un ritmo más pausado, son menos la cantidad de actividades o proyectos que realizar y hay un contacto presencial con docente y estudiantes. Como puntos débiles se encuentra el desplazamiento que por situación laboral se imposibilita para tutorías o entrega de trabajos y que no se abren tutorías en todos los Centros Universitarios de la institución.

De la modalidad híbrida les gusta la combinación que esta tiene (aunque es la de menor preferida), porque se tiene la interacción en la plataforma y la aplicación de exámenes para medir el conocimiento, favorece el que no se hacen tantas actividades en línea y hay acceso a docentes para consultar dudas. Lo que no les gusta a algunas personas son precisamente la aplicación de pruebas escritas, tendencia a confundir fechas de entrega de actividades y las de aplicación de exámenes.

En la modalidad $100 \%$ virtual, el estudiantado cita que se aprende mucho más, al no realizarse exámenes se favorece la investigación (en algunas asignaturas), no se depende de un horario para realizar las actividades, aunque sí de una fecha y hora de entrega, permite organizarse mejor con otras labores que se lleven a cabo; el punto débil está relacionado con que no siempre hay acceso a internet, no se descarguen bien las lecturas o estas sean muchas y muy pesadas para usar el teléfono celular para ello, así como al hecho de que teniendo la facilidad de consultar por la plataforma, las respuestas de docentes son en ocasiones lentas.

Por otra parte, la totalidad de estudiantes manifiesta la importancia y la necesidad de hacer mejoras en las asignaturas con modalidades híbridas y 100\% virtuales, que se acoplen a las necesidades particulares de ellos y ellas como estudiantes que trabajan en horarios poco convencionales, en los que el estrés propio de exponer sus vidas al servicio de la protección e integralidad de otras personas cobra un estrés mayor en sus vidas, ello con el fin no solo de garantizar un mejor aprendizaje sino de apoyar el logro de los objetivos de los cursos y el éxito en el rendimiento académico.

Entre las sugerencias que citan están:

- Utilizar como complemento la posibilidad de videoconferencias, teletutorías y programas de radio a través de ONDAUNED, ya que estos, al estar grabados, pueden escucharse en cualquier momento y desde cualquier lugar.

- Tomar en cuenta los tiempos de entrega de trabajos o proyectos extensos, considerando la situación laboral que la mayoría de estudiantes de las Carreras de Ciencias Criminológicas y Ciencias Policiales tienen.

- Mayor compromiso por parte de docentes para revisar trabajos, brindar realimentación y entregar calificaciones en tiempos razonables. 
- Utilizar materiales y lecturas actualizadas y adecuadas a la realidad nacional, de forma tal que se pueda visualizar la situación país ante una determinada problemática.

- Brindar instrucciones claras al estudiantado de lo que se les solicita en los trabajos, proyectos y actividades de la plataforma.

Es importante señalar que estas sugerencias coinciden, a su vez, con las expuestas por García (2015) cuando este menciona:

Si el estudiante universitario es a la vez asalariado y ha tenido una baja calidad en la educación preuniversitaria, la percepción docente señala que se espera que tenga un bajo rendimiento académico, influencia negativa que se podría reducir si se logra una mejora en el acceso al campus universitario y si se elevan las capacidades docentes para mantener la atención del estudiantado y promover un aprendizaje efectivo. (p. 13)

Por ende, uno de los retos principales que deben asumir las personas encargadas de las Carreras de Ciencias Policiales y Ciencias Criminológicas y de las Cátedras que les prestan servicios es que debe existir en primera instancia una sensibilización hacia la situación particular de esta población estudiantil para, posteriormente, desarrollar ajustes en la metodología de enseñanzaaprendizaje y en el uso de las tecnologías educativas, a fin de proveerle apoyos articulados que ayuden en la aprehensión de conocimientos y en la mejoría del rendimiento académico.

\section{Discusión y conclusiones}

Se considera alcanzado el objetivo planteado con la investigación, en el tanto que se logra determinar la incidencia del uso de la plataforma educativa en línea para el aprendizaje de estudiantes de asignaturas de las carreras de Ciencias Criminológicas y Ciencias Policiales, así como las fortalezas y deficiencias que, en este sentido, según la precepción de estudiantes, tiene.

Se destaca el hecho de la importancia de la educación a distancia para personas que presentan dificultades horarias que les impiden acceder a la educación superior, de ahí que se privilegien las plataformas educativas en línea para alcanzar este fin.

El estudiantado en esta modalidad de enseñanza aprendizaje se torna en personas que desarrollan habilidades y metacogniciones que les permiten el aprendizaje autónomo y autorregulado, eso sí, con una adecuada mediación pedagógica en la que logren un acercamiento con docentes y estudiantado para satisfacer dudas y consultas mediante una comunicación bidireccional sincrónica y asincrónica, según sea el caso. 
doi: http://dx.doi.org/10.15359/ree.22-1.2

URL: http://www.una.ac.cr/educare

CORREO: educare@una.cr

Tal y como lo presenta el estudiantado en la investigación, tanto el uso de las TIC como la educación a distancia requieren de una adecuada mediación pedagógica por parte del profesorado tutor que le permita, al estudiantado, encontrarle sentido a lo que se estudia, a la vez que se le desarrolle potencialidades requeridas para el análisis y comprensión de la información transmitida.

Es así cómo, el uso de las TIC se convierte en la herramienta funcional para comprender, de mejor manera, el aprendizaje que se desea obtener en el estudiantado, ya que la diversidad de estrategias de mediación favorece los procesos educativos siempre y cuando estén diseñadas para fines académicos y exista una adecuada planificación en estas mismas, encaminada a favorecer competencias profesionales y la aprehensión de conocimientos.

Así mismo, se pueden colegir aspectos importantes referentes a las asignaturas con apoyo virtual, para que estas sean en realidad un aporte para el éxito académico estudiantil.

Se hace necesario que tanto docentes como personal encargado de las Carreras de Ciencias Policiales y de Ciencias Criminológicas y de las diferentes cátedras que ofertan asignaturas se sensibilicen acerca de las particularidades de estudiantes que trabajany estudian simultáneamente, a fin de que los diseños curriculares y las metodologías de enseñanza aprendizaje sean adecuadas y óptimas para favorecer su crecimiento profesional y rendimiento académico.

A su vez, se puede inferir la necesidad de mayor contacto e interacción entre docente y estudiantes que favorezca el aprendizaje (Barberà, Badia \& Mominó, 2001), con aspectos como que las consultas sean respondidas con prontitud, ya que no siempre tienen acceso a internet para conocer un determinado detalle de la asignatura o de la actividad que deben entregar en una fecha definida, que se les apoye y oriente hacia procesos de autoaprendizaje. De igual manera se nota la necesidad de reforzar la parte práctica, es decir, que la teoría se lleve a cabo y se ejecute, así como actividades que promuevan la investigación, ya que al finalizar los estudios denotan vacíos formativos en esta área.

Por otro lado, si bien aprecian las ventajas de las asignaturas apoyadas en plataformas virtuales, se nota la necesidad de mayor contacto con su docente, ya sea con al menos una tutoría o teletutoría, o el uso de TIC adicionales, ya que esto les facilitaría la comprensión de los contenidos temáticos y una explicación más detallada de lo que se les solicita en las actividades o proyectos de la asignatura.

Así mismo es fundamental una mayor capacitación a docentes sobre el uso de la plataforma, tipos de actividades dinámicas y creativas, así como sobre las posibilidades de mediación pedagógica que esta brinda, tanto en aspectos formales del desarrollo de las asignaturas como de contenidos, así como de una adecuada atención y mediación, con el fin de que la plataforma no se torne en una herramienta tecnológica sin potencial de aprendizaje, sino que, por el contrario, fomente verdaderos aprendizajes en estos entornos. 
De esta forma se pueden establecer las siguientes conclusiones:

1. Se logró verificar que el uso de plataformas educativas en línea, siempre que este recurso esté apoyado por una adecuada mediación e interacción con quien ejerce la docencia, favorece el proceso de aprendizaje en el estudiantado encuestado de las carreras de Ciencias Criminológicas y Ciencias Policiales, pertenecientes a la Escuela de Ciencias Sociales y Humanidades de la UNED, que son parte de un modelo educativo a distancia.

2. Del estudio se desprende que el estudiantado sí encuentra diferencias para su aprendizaje con el uso de la tecnología en aspectos como: material actualizado; interacción entre compañeros y compañeras que favorece la construcción de un aprendizaje continuo, permanente y reflexivo; hay innovación en los procesos de aprendizaje, y las asignaturas en línea son más participativas, dinámicas y creativas.

2. El estudiantado que participó en el estudio manifiesta estar de acuerdo con el uso de la tecnología al privilegiar la modalidad $100 \%$ virtual sobre la tradicional y la híbrida, ya que reciben una mejor mediación pedagógica a través de la plataforma, lo cual se acompaña del intercambio entre docente y estudiantes y entre iguales, lo que favorece los procesos de enseñanza aprendizaje.

3. Sin embargo, el estudiantado es crítico al mencionar que al personal docente le falta mayor diversificación, actualización y preparación en el uso de la plataforma y en las actividades formativas y sumativas que emplean, menor tiempo de respuesta ante consultas, mayor disponibilidad para apoyarles, tutoriales y consignas más claras, que se promueva la investigación, uso de materiales y lecturas actualizadas y pertinentes, así como realizar ejercicios de autoevaluación para mejorar la asignatura la próxima vez que se oferte.

4. Se hace indispensable en las asignaturas de las Carreras de Ciencias Criminológicas y Ciencias Policiales considerar el factor de riesgo y estrés que el estudiantado experimenta en el desempeño de sus funciones laborales, situación que indudablemente va a repercutir en su aprovechamiento académico y formativo. Se requiere de docentes que conozcan la realidad del estudiantado y la cotidianidad a la que se enfrentan como estudiantes a distancia, personas trabajadoras e integrantes y responsables de una familia.

5. En síntesis, se puede afirmar que el uso de la tecnología y el apoyo en plataformas virtuales en las asignaturas de las carreras en las cuales se realizó el estudio son una fortaleza para el proceso de aprendizajes del estudiantado, pero se requiere una optimización de los recursos y de los procesos de aprendizaje, una mediación pedagógica más adecuada a las necesidades particulares de esta población en la que el uso de otras herramientas como videoconferencias, teletutorías y programas de radio (entre otras) les apoyen; así como reforzar la capacitación para docentes en sistemas de evaluación, aprovechamiento de la tecnología para el aprendizaje y mediación pedagógica. 
doi: http://dx.doi.org/10.15359/ree.22-1.2

URL: http://www.una.ac.cr/educare

CORREO: educare@una.cr

\section{Referencias}

Axelbred, M., \& Valle, J. (1981). Stress control program for police officers in the City of Miami Police Department (From stress and police personnel). In L. Territo, y H. J. (Eds.), Vetter. Stress and Police Personnel (pp. 279-286). Boston: Allyn \& Bacon.

Barberà, E. (Coord.), Badia, A. Mominó, J. M. (2001). La incógnita de la educación a distancia. Barcelona: Horsori.

Brown, J. M., \& Campbell, E. A. (1994). Stress and policing: Sources and strategies. Chichester:Wiley. doi: https://doi.org/10.1080/10439463.1994.9964703

Carrillo, S. y Ríos, J. G. (2013). Trabajo y rendimiento escolar de los estudiantes universitarios. El caso de la Universidad de Guadalajara, México. Revista de la Educación Superior, 42(166), 9-34, Recuperado de http://www.scielo.org.mx/pdf/resu/v42n166/v42n166a1.pdf

Clark, R. E. (2001). A summary of the disagreements. With the "mere vehicles" argument. En C. Schlosser \& M. Simonson (Eds.), Learning from media. Arguments, analysis, and evidence (pp. 125-136). Greenwich: Information Age publishing.

Comisión Europea (30 de diciembre de 2006). Recomendación del parlamento europeo y del consejo del 18 de diciembre de 2006 sobre las competencias clave para el aprendizaje permanente. Diario Oficial de la Unión Europea, 394, pp. 10-18. Recuperado de http://eurlex.europa.eu/legal-content/ES/TXT/PDF/?uri=CELEX:32006H0962\&from=ES

Cooper, C. L., Davidson, M. J. y Robinson, P. (1982). Stress in the police service. Journal of Occupational Medicine, 24(1), 30-36.

Cooperberg, A. F. (2002). Las herramientas que facilitan la comunicación y el proceso de enseñanza-aprendizaje en los entornos de educación a distancia. Revista de Educación a Distancia, 3, 1-35. Recuperado de http://revistas.um.es/red/article/view/25211/24491

Dans, E. (2009). Educación online: Plataformas educativas y el dilema de la apertura. Revista de Universidad y Sociedad del Conocimiento, 6(1), 21-30. Recuperado de http://www.redalyc. org/articulo.oa?id=78011179010

García-Aretio, L. (1987). Hacia una definición de educación a distancia. Boletín informativo de la Asociación Iberoamericana de Educación Superior a Distancia, 4(18), 1-9. Recuperado de http://www2.uned.es/catedraunesco-ead/articulos/1987/hacia\%20una\%20definicion\%20 de\%20educacion\%20a\%20distancia.pdf

García-Aretio, L. (2001). La educación a distancia. De la teoría a la práctica (2ª ed.). España: Editorial Ariel. 
García-Aretio, L. (2014). La educación a distancia. Bases conceptuales. Contextos universitarios mediados, 14(7).

García, E. R. (2015). Percepción docente del rendimiento académico de los estudiantes universitarios de la USAC Guatemala que trabajan y estudian. Innovaciones Educativas, 17(22), 5-14. doi: https://doi.org/10.22458/ie.v17i22.741

Hernández, R., Fernández, C. y Baptista, P. (2014). Metodología de la Investigación. México: MacGraw-Hill.

Instituto Nacional para la Seguridad y Salud Ocupacional (NIOSH). (2015). El estrés en el trabajo. NIOSH, 99-101, 1-32. Recuperado de http://www.cdc.gov/spanish/niosh/docs/99-101 sp/

Kroes, W.H. (1985). Society's victim: the police officer. Springfield, IL: Charles Thomas.

Lamas, H. (2008). Aprendizaje autorregulado, motivación y rendimiento académico. Liberabit, 14, 15-20. Recuperado de http://www.scielo.org.pe/scielo.php?pid=S1729$\underline{48272008000100003 \& \text { script }=\text { sci arttext }}$

Manrique, L. (23 de marzo al 4 de abril de 2004). El aprendizaje autónomo en la educación a distancia. Debates de educación (Barcelona). Ponencia presentada en Primer Congreso Virtual Latinoamericano de Educación a Distancia. Pontificia Universidad Católica del Perú, Perú. Recuperado de http://departamento.pucp.edu.pe/educacion/noticias/elaprendizaje-autonomo-en-la-educacion-a-distancia/

Monereo, C. (Coord.). (2008). Ser estratégico y autónomo aprendiendo. Unidades didácticas de enseñanza estratégica para la ESO. Barcelona: Graó.

Monereo, C. y Barberá, E. (2000). Diseño instruccional de las estrategias de aprendizaje en entornos educativos no-formales. Madrid, España: Ediciones de la Universidad Oberta de Catalunya.

Pagano, C. M. (2007). Los tutores en la educación a distancia. Un aporte teórico. Revista de universidad y sociedad del conocimiento, 4(2), 1-11. Recuperado de http://www.uoc.edu/ rusc/4/2/dt/esp/pagano.pdf

Pintrich, P. R. (2000). The role of goal orientation in self-regulated learning. En M. Boekaerts, P. R. Pintrich \&, M. Zeidner (Eds.), Handbook of self-regulation (pp. 451-502). California: Academic Press. doi: https://doi.org/10.1016/B978-012109890-2/50043-3

Piñeiro, I., Valle, A., González, R., y Rodríguez, S. (2002). Autorregulación del aprendizaje y estrategias de estudio. En J. A. González-Pienda, J. C. Núñez, L. Álvarez y E. Soler (Coords.), Estrategias de aprendizaje. Concepto, evaluación e intervención (pp. 17-38). Madrid: Pirámide. 
doi: http://dx.doi.org/10.15359/ree.22-1.2

URL: http://www.una.ac.cr/educare

CORREO: educare@una.cr

Reese, J. T. (1986). Policing the violent society: The American experience. Stress Medicine, 2, 233240. doi: https://doi.org/10.1002/smi.2460020309

Rodríguez, R. M. (2010). El impacto de las TIC en la transformación de la enseñanza universitaria: Repensar los modelos de enseñanza y aprendizaje. TESI. Teoría de la Educación y Cultura en la Sociedad de la Información, 11(3), 32-68. Recuperado de http://gredos.usal.es/jspui/ bitstream/10366/72850/1/EL IMPACTO DE LAS TIC EN LA TRANSFORMACI.pdf

Sánchez, J. (2009). Plataformas de enseñanza virtual para entornos educativos. Pixel-Bit. Revista de Medios y Educación, 34, 217-233. Recuperado de http://acdc.sav.us.es/ojs/index.php/ pixelbit/article/view/725/607

Smith, B. y Álvarez, M. (2007). Revictimización un fenómeno invisibilizado en las instituciones. Medicina Legal Costa Rica, 24(1), 65-101. Recuperado de http://www.scielo.sa.cr/scielo. php?script=sci arttext\&pid=S1409-00152007000100004

Universidad Estatal a Distancia (UNED). (2016). Estadística de matrícula período 2015 (documento CIEI003-2016).San José, CostaRica:CIEI,UIAE.Recuperado de http://www.uned.ac.cr/viplan/ images/ciei/estadisticas/INFORME MATRICULA ANUAL 2015/Estad\%C3\%ADsticas de matr\%C3\%ADcula Per\%C3\%ADodo 2015.pdf

Universidad Estatal a Distancia, Consejo Universitario. (2004). Modelo pedagógico. San José: Autor. Recuperado de https://www.uned.ac.cr/conuniversitario/images/docs cu/ politicas academicas/ModeloPedagogico 000.pdf

Zimmerman, B. J. (2002). Becoming a self-regulated learner: An overview. Theory into practice, 41(2), 64-70. doi: https://doi.org/10.1207/s15430421tip4102 2 